\title{
A maternal deletion upstream of the imprint control region 2 in 11p15 causes loss of methylation and familial Beckwith-Wiedemann syndrome
}

\author{
Jasmin Beygo $^{1}$, Ivana Joksic ${ }^{2}$, Tim M Strom ${ }^{3}$, Hermann-Josef Lüdecke ${ }^{1}$, Julia Kolarova ${ }^{4}$, Reiner Siebert ${ }^{4}$, \\ Zeljko Mikovic ${ }^{2}$, Bernhard Horsthemke ${ }^{1}$ and Karin Buiting ${ }^{\star, 1}$
}

Beckwith-Wiedemann syndrome (BWS; OMIM \#130650) is an overgrowth syndrome caused by different genetic or epigenetic alterations affecting imprinted regions on chromosome $11 \mathrm{p} 15.5$. Here we report a family with multiple offspring affected with BWS including giant omphalocoeles in which maternal transmission of a chromosomal rearrangement including an inversion and two deletions leads to hypomethylation of the imprint control region 2 (ICR2). As the deletion includes the promoter and $5^{\prime}$ part of the KCNQ1 gene, we suggest that transcription of this gene may be involved in establishing the maternal methylation imprint of the ICR2, which is located in intron 10 of KCNQ1.

European Journal of Human Genetics (2016) 24, 1280-1286; doi:10.1038/ejhg.2016.3; published online 3 February 2016

\section{INTRODUCTION}

Beckwith-Wiedemann syndrome (BWS; OMIM \#130650) is an overgrowth syndrome caused by different genetic or epigenetic disturbances affecting the imprinted regions on chromosome 11p15.5. The disease is characterised by macrosomia, macroglossia, abdominal wall defects, visceromegaly and an elevated risk for childhood tumours, although the phenotypic presentation can be highly variable. ${ }^{1}$

The chromosomal region 11p15.5 harbours two imprinted domains, each of which is regulated by its own imprinting control region (ICR): the telomeric ICR1 and the centromeric ICR2 (new terminology IGF2/H19 intergenic differentially methylated region (DMR) and KCNQ1OT1 TSS DMR, respectively, according to EUCID; www. imprinting-disorders.eu/). The ICR2 controls the parent-of-originspecific expression of several genes including the maternally expressed genes CDKN1C (cyclin-dependent kinase inhibitor 1 C), SLC22A18 (solute carrier family 22, member 18) and KCNQ1 (potassium channel, voltage-gated KQT-like subfamily $Q$, member 1 ), as well as its paternally expressed antisense transcript KCNQ1OT1 (KCNQ1 opposite strand/ antisense transcript 1). It spans the $5^{\prime}$ end of KCNQ1OT1 within intron 10 of KCNQ1 and is methylated on the maternal allele only. Hypomethylation of the ICR2 is the most common molecular cause of BWS and is present in about $50-60 \%$ of the cases. ${ }^{2}$ It leads to the loss of expression of the maternally expressed genes including the growth inhibitor CDKN1C and a biallelic expression of the paternally expressed genes. In the majority of cases, the hypomethylation is present in a mosaic state with a highly variable degree of methylation and occurs sporadically as a postzygotic event. ${ }^{3,4}$ Other molecular causes of BWS include hypermethylation of the ICR1 ( 5-10\%), paternal uniparental disomy of chromosome 11p15 ( 20-25\%), paternal duplications $(\sim 1-2 \%)$ and point mutations in CDKN1C $(\sim 5 \%))^{2,5}$ So far, only a few familial BWS cases caused by deletions or duplications affecting the centromeric imprinted region on $11 \mathrm{p} 15.5$ have been described in the literature (reviewed in Begemann et al., ${ }^{6}$ Baskin et al. ${ }^{7}$ and Gurrieri et al. ${ }^{8}$ ). Although varying greatly in size, all deletions and all but one duplication include the ICR2 itself.

In 2004, Niemitz et al. ${ }^{9}$ described the first individual with BWS caused by a deletion of the centromeric imprinted domain on $11 \mathrm{p} 15$ (Figure 1). The deletion had a size of about $250 \mathrm{~kb}$ and spanned a large part of the KCNQ1 gene including its antisense transcript KCNQ1OT1 and the ICR2. A similar deletion of $\sim 330 \mathrm{~kb}$ also abolishing the ICR2 has been reported a few years later. ${ }^{10}$ Another deletion encompassing again about $330 \mathrm{~kb}$ within the KCNQ1 gene and abolishing the ICR2 has been described by Baskin et al. ${ }^{7}$ They also identified a second, much smaller deletion spanning only about $0.9 \mathrm{~kb}$, but deleting a large part of the ICR2 as well. Another BWS patient with a mild phenotype together with cardiac problems has a $198 \mathrm{~kb}$ deletion that removes the $3^{\prime}$ part of KCNQ1 including the ICR $2 .{ }^{8}$ Zollino et al. ${ }^{11}$ described the so far largest deletion, which spans about $900 \mathrm{~kb}$. This is also the only deletion that not only removes the ICR2 but also the CDKN1C gene itself. Last, Demars et al. ${ }^{12}$ described a duplication of about $50 \mathrm{~kb}$ within the KCNQ1 gene that does not overlap with the ICR2, but leads to hypomethylation of the ICR2 and BWS.

We here report on a familial case of BWS with a complex chromosomal rearrangement that does not include the ICR2, but leads to hypomethylation of the ICR2 after maternal transmission.

\section{MATERIALS AND METHODS}

Samples

Peripheral blood samples from the parents (II-1 and II-2; Figure 2) and the maternal grandparents (I-1 and I-2), as well as chorionic villi (III-5 and III-6), amniotic fluid and umbilical cord blood (III-6; 22nd week of gestation), were

${ }^{1}$ Institut für Humangenetik, Universitätsklinikum Essen, Universität Duisburg-Essen, Essen, Germany; ${ }^{2}$ Clinic of Gynecology and Obstetrics Narodni front, Belgrade, Serbia; ${ }^{3}$ Institut für Humangenetik, Technische Universität München, München, Germany; ${ }^{4}$ Institut für Humangenetik, Christian-Albrechts-Universität Kiel and Universitätsklinikum Schleswig-Holstein, Campus Kiel, Germany

${ }^{*}$ Correspondence: Dr K Buiting, Institut für Humangenetik, Universitätsklinikum Essen, Universität Duisburg-Essen, Hufelandstrasse 55, 45147 Essen, Germany. Tel: +49 201 723 4555 ; Fax: +49 201723 5900; E-mail: Karin.Buiting@uni-due.de

Received 17 September 2015; revised 15 December 2015; accepted 22 December 2015; published online 3 February 2016 




Figure 1 Deletions affecting the ICR2. The scheme shows part of the centromeric imprinted region with the ICR2 and the deletions reported in the literature and here so far. Additionally, a 50 kb duplication within KCNQ1 is given in grey. Not drawn to scale.



Figure 2 Family pedigree. The mother (II-2) is a healthy carrier of the rearrangement, which is located on her paternal allele. She has transmitted it (presumably) to all six children. The grey colour for III-3 (spontaneous abortion) indicates that no phenotype data were available, but a disease state is assumed.

obtained after written informed consent. The study was approved by the ethics committee of the University Duisburg-Essen. Information regarding this family has been submitted to the LOVD (patient ID 0000054752; http://databases.lovd. $\mathrm{nl} /$ shared/individuals).

\section{DNA extraction}

DNA was extracted using the Flexigene Kit (Qiagen, Hilden, Germany) following the manufacturer's protocol for the different tissue types. Maternal contamination was excluded in all fetal samples using the Powerplex 16 Kit (Promega, Mannheim, Germany) before the subsequently described analyses.

\section{Bisulphite conversion}

The bisulphite conversion of an umbilical cord blood genomic DNA sample (III-6) was performed using the EZ-DNA Methylation Kit (Zymo Research Corporation, Irvine, CA, USA).

\section{Methylation-specific multiplex ligation-dependent probe amplification}

Gene dosage and methylation analyses of the chromosomal region 11p15 including the two ICRs were carried out using the SALSA MLPA Kit ME030-C3 (MRC Holland, Amsterdam, The Netherlands) according to the manufacturer's manual. Amplification products were analysed on an ABI3130XL capillary sequencer (Applied Biosystems, Darmstadt, Germany) followed by data analysis with the GeneMarker Software (Softgenetics, State College, PA, USA). The methylation-specific multiplex ligation-dependent probe amplification (MS-MLPA) Kits ME032-A1 and ME028-B2 including imprinted regions on chromosomes $6,7,14$ and 15, respectively, were processed similarly.

\section{Microsatellite analysis}

Microsatellite analysis (MSA) for chromosome 11 was performed using 11 markers for the chromosomal region 11p15.5 to 11q25 (D11S1984, D11S2362, D11S1999, D11S1981, D11S1392, D11S2000, D11S1986, D11S1998, D11S4464, D11S912, D11S968) using standard protocols. Fluorescence-tagged PCR products were analysed on an ABI3130XL capillary sequencer (Applied Biosystems) and the GeneMarker software (Softgenetics).

\section{SNP array analysis}

Genome-wide copy number analysis was performed using the Affymetrix CytoScan HD array (Affymetrix, Santa Clara, CA, USA) following the manufacturer's instructions. The Chromosome Analysis Suite (Chas) software (v.2.1.0.16; Affymetrix) was used for data analysis.

\section{Array-based methylation analysis}

Array-based methylation analysis on DNA derived from cord blood of individual III-6 was performed in duplicate applying the Infinium HumanMethylation450 BeadChip (Illumina, San Diego, CA, USA) as described recently. ${ }^{13}$ The DNA methylation of the individual III-6 was compared with the DNA methylation determined in peripheral blood DNA of a previously published control cohort $(n=19) .{ }^{13}$ The raw hybridisation signals were analysed with the GenomeStudio software (version 2011.1; Methylation Analysis Module version 1.9.0; Illumina) applying the default settings and internal controls for normalisation. Loci with a detection $P$-value $\geqslant 0.01$ in at least one sample were excluded. The evaluation focused on the 891 tag CpGs shown by Court et al. ${ }^{14}$ to be imprinted, from which 884 passed the quality control filters. The mean DNA methylation value (AVG- $\beta$ values) of the two 
technical replicates of III- 6 was calculated for these 884 CpG and compared with the mean DNA methylation values of the control cohort.

First, the normal DNA methylation range for each CpG locus was defined as the mean DNA methylation of the controls \pm 3 SDs. Second, the difference between the mean DNA methylation of the case III-6 and the mean DNA methylation of the control cohort was determined. An aberrant methylated CpG locus in III-6 was defined as having a mean DNA methylation value outside the normal DNA methylation range as well as showing a difference between the mean DNA methylation of III- 6 and the controls $>$ I0,3I.

\section{Whole-genome sequencing}

Whole-genome sequencing (WGS) was conducted in the mother (II-2) as described previously. ${ }^{15}$ Briefly, the library was prepared using PCR-free protocols (TruSeq DNA PCR-Free; Illumina) and sequencing was performed on the HiSeq2000/2500 systems (Illumina).

\section{Breakpoint verification}

For verification of the breakpoints, specific primers were designed based on the WGS data. Amplification of the junction fragments was conducted using standard protocols. For primer sequences and PCR conditions, see Supplementary Table S1. Sequencing of PCR products was carried out on an ABI3130XL capillary sequencer (Applied Biosystems). Sequence analysis was performed using the Sequencing Analysis (Applied Biosystems) and the Geneious software (Biomatters, Auckland, New Zealand).

\section{RESULTS}

\section{Clinical findings}

A 24-year-old pregnant woman was referred to genetic counselling in her fifth pregnancy because at least three of the four previous pregnancies were affected by omphalocoeles (Figure 2). The woman and her partner were healthy Caucasians from southern Serbia and non-consanguineous and had no living children. Their family history was unremarkable. A conventional cytogenetic analysis yielded a normal karyotype in both parents $(46, \mathrm{XX}$ and $46, \mathrm{XY})$. The first two pregnancies (III-1 and III-2) of the couple were terminated owing to the presence of a large omphalocoele in the fetus and poor postnatal prognosis at 18th gestational week (g.w.) and 15th g.w., respectively. The third pregnancy (III-3) ended as a missed abortion in early first trimester and no phenotypic data is available for this child. During the fourth pregnancy (III-4), at level II ultrasound examination in the second trimester, again an omphalocoele was detected, but the parents decided to continue the pregnancy. Amniocentesis was performed and yielded a normal female karyotype $(46, \mathrm{XX})$. No additional anomalies were described. The pregnancy was complicated with premature rupture of fetal membranes and delivery at 30th g.w. (birth weight was $1800 \mathrm{~g}$, 97th percentile, Apgar score at $1^{\prime} 5$ ). After birth, the baby developed severe respiratory distress and died of complications 4 days later.

The fifth pregnancy of the couple started as a dizygotic twin pregnancy. Ultrasound examination at $13+1$ g.w. revealed different abnormalities in both twins. The first twin (III-5) showed borderline nuchal translucency thickness $(3 \mathrm{~mm})$, reverse a-wave in ductus venosus, bilateral pyelectasis, megacystis and omphalocoele (Supplementary Figure S1). In the second twin (III-6), only an omphalocoele was noted, nuchal transluscency was $1.4 \mathrm{~mm}$ and other first trimester ultrasound markers of aneuploidy were negative (Supplementary Figure S1). The fetal biometry of both twins was appropriate for gestation age. Interestingly, both placentas were thick ( 36 and $20 \mathrm{~mm}$ ) with numerous microcystic changes, which were clinically suggestive of placental mesenchymal dysplasia. Chorionic villus sampling was performed for cytogenetic analysis, which showed a normal karyotype in both twins (III-5 46,XY, III-6 46,XX). Intrauterine death of the first fetus occurred at the 14th g.w. Level II ultrasound examination at 22nd g.w. of the second twin confirmed the presence of a large omphalocoele (containing liver and small bowel) and placental mesenchymal dysplasia; no additional anomalies were noted. On parents request and due to poor postnatal prognosis, the pregnancy was terminated at 22nd g.w. The presence of an omphalocoele and placental mesenchymal dysplasia led to the tentative clinical diagnosis of BWS.

\section{Molecular findings}

To verify the initial clinical diagnosis of BWS, molecular genetic analysis was performed using MS-MLPA of chromosome 11p15 in both affected twins (III-5 and III-6; Figure 2). The results showed a normal methylation at the ICR1, but a complete loss of methylation at the ICR2 for all four methylation-sensitive probes (grey shaded probes in Supplementary Table S2). Gene dosage was normal for all investigated probes on chromosome $11 \mathrm{p} 15.5$, with the exception of one probe (L02903) located in the $5^{\prime}$ region of KCNQ1, $\sim 237 \mathrm{~kb}$ upstream of the ICR2. At this site we observed a reduced dosage of $50 \%$, indicating the presence of a heterozygous deletion. The same reduction could be observed in the mother (II-2), while the methylation was normal at both ICRs. MS-MLPA results for the father (II-1) and the maternal grandparents (I-1 and I-2) showed normal methylation patterns and gene dosage for all probes. No material was available from the previous pregnancies. Taken together, the results of the MLPA analyses confirmed the clinical diagnosis of BWS.

As patients with a clinical presentation of BWS and Silver-Russel syndrome sometimes harbour multilocus imprinting disturbances, ${ }^{16}$ we tested the methylation status of the twins by further MS-MLPA analyses for imprinted loci on chromosomes 6, 7, 14 and 15 (III-5 and III-6) but no aberrant methylation could be detected for the loci studied (data not shown). Furthermore, array-based DNA methylation analyses were conducted in one of the twins (III-6). These analyses revealed aberrant DNA methylation values (defined as mean DNA methylation values in III-6 exceeding the mean DNA methylation in controls \pm 3 SDs with an absolute difference of the means of at least 0.3 ) for $30 / 30 \mathrm{CpG}$ loci in the gene KCNQ1/KCNQ1OT1 corroborating the MLPA results. From the remaining 854 tag CpGs of imprinted loci analysed, only one met the statistical threshold for aberrant methylation. This CpG was 1/8 tag CpGs representing the gene RGMA (Supplementary Table S3).

Parental inheritance of chromosome 11 was investigated by MSA. Three informative markers for the short arm of chromosome 11 indicated that the twins inherited different paternal, but the same maternal allele. Strikingly, for the most distal marker in $11 \mathrm{p} 15.5$ (D11S1984), which is located about $1 \mathrm{Mbp}$ telomeric to KCNQ1, only a paternal contribution was detectable in both twins, indicating the presence of a second heterozygous deletion in addition to the one already detected by MLPA (Figure 3). Indeed, using the Affymetrix CytoScan array (Affymetrix) two deletions spanning roughly 285 and $115 \mathrm{~kb}$, respectively, could be detected (Supplementary Figure S2; deletion sizes differ from Sanger results because of the distribution of probes used by the array). The telomeric deletion includes the MSA marker, whereas the centromeric one includes the MLPA probe in the $5^{\prime}$ region of KCNQ1. Two deletions in such a close proximity to each other are often indicative of a more complex rearrangement. As a previously performed cytogenetic analysis had yielded a normal result, WGS was carried out in the mother. The analysis of the WGS data verified the presence of both deletions and additionally showed an inversion of the region in between. In addition, the results indicated as MLPA and SNP array data already had - the presence of one normal copy of chromosome 11 without deletions and inversion. 


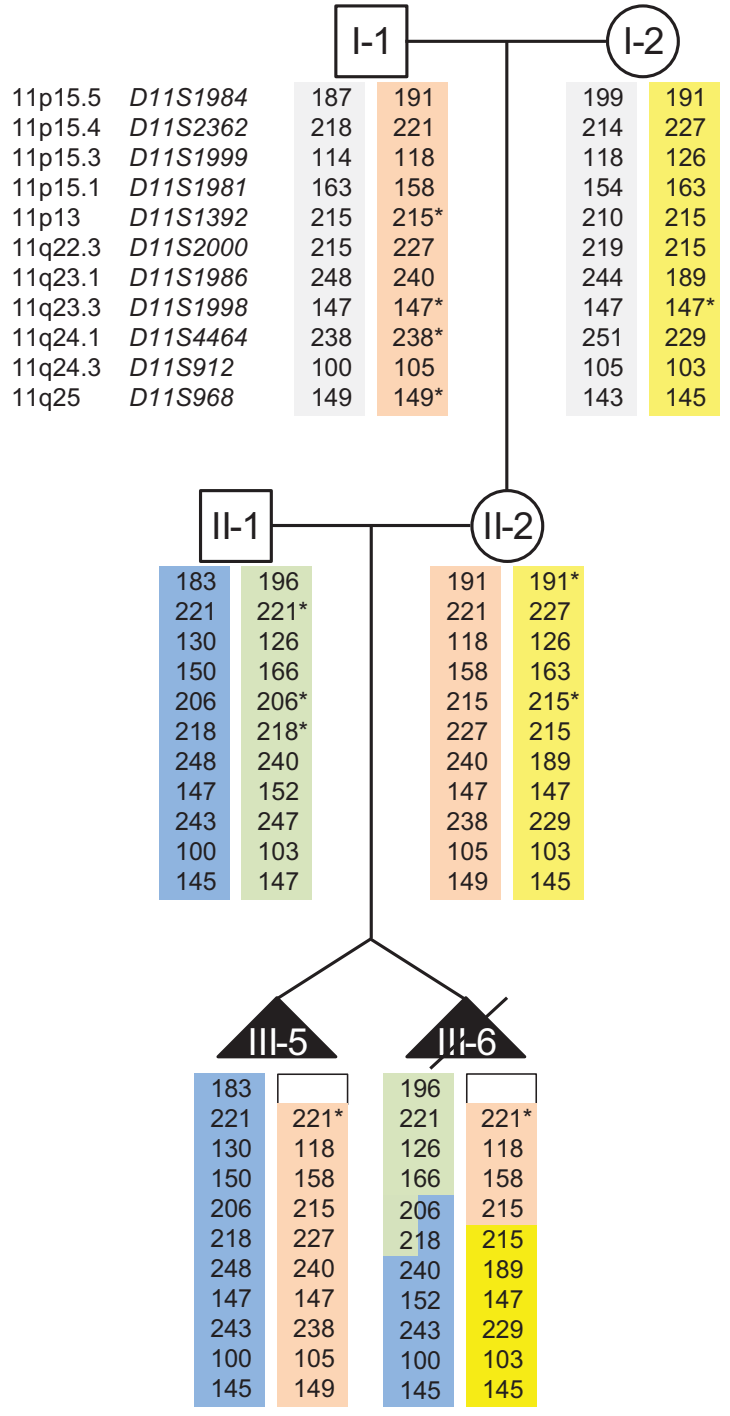

Figure 3 Results of the microsatellite analysis on chromosome 11. MSA results for the 11 investigated markers spread along chromosome 11 . Parental origin is indicated by colours. For III-5 and III-6 paternal contribution could only be detected for one marker (D11S1984, 11p15.5). For the short arm of chromosome 11, the twins have inherited different paternal but the same maternal allele. For individual III-6 two crossing over events, one on the paternal and one on the maternal allele, were observed. The breakpoints on the paternal allele cannot be clearly denoted as the results for two markers (D11S1392 and D11S2000) can be assigned to both alleles. This is indicated by a two colour scheme. For the maternal allele, the event took place between the last marker for the short and the first marker for the long arm of the chromosome. Asterisk indicates a hemi- or homozygous state, which is not distinguishable by this method.

Verification of the deletion breakpoints was performed by PCR and subsequent sequencing of the junction fragments. The results revealed a complex nature of the rearrangement including the two deletions, the inversion and several insertions of smaller fragments both inverted and not inverted and originating mainly from chromosome $11 \mathrm{p} 15$ (Figure 4). The mother is a healthy carrier of the rearrangement, which is located on her paternal chromosome and which she has passed on to both twins and presumably to her other affected children as well (Figure 2). As no evidence for this rearrangement was detectable in the maternal grandfather, it seems to have arisen de novo.
As determined by Sanger sequencing, the telomeric deletion spans 298.042 kb (hg19, chr11:g.1565895_1863936del), overlaps with the MSA marker D11S1984 and includes 14 genes: DUSP8, KRATAP5-AS1, KRATAP5-1-6, FAM99A and B, IFITM10, CTSD, SYT8 and TNNI2 (telomeric to centromeric orientation; Figure $5 \mathrm{a}$ ). The smaller centromeric deletion spans 120.719 or $120.724 \mathrm{~kb}$ owing to a $5 \mathrm{bp}$ overlap (hg19, chr11:g.2368677_2489400del) and includes five genes: the 5' part of CD81-AS, CD81, TSSC4 and TRPM5, all of which are biallelically expressed and not imprinted. For TRPM5, which has previously been reported to be imprinted, we investigated its imprinting status in blood of two healthy individuals and found no evidence for monoallelic expression (for details see Supplementary Information). In addition, the deletion includes the $5^{\prime}$ region of the imprinted gene KCNQ1 but not the ICR2, which is aberrantly methylated in both twins (telomeric to centromeric orientation; Figure 5a). The deletion abolishes the start exons of both transcript variants of KCNQ1 and a large part of intron 1 (exon 1 of NM_000218.2 and CCDS7736.1 and the alternative exon 1 of NM_181798.1; equals exons $1 \mathrm{a}$ and $1 \mathrm{~b}$ according to the numbering by Cerrato et al. ${ }^{17}$ and Neyroud et al.; ${ }^{18}$ Figure $\left.5 b\right)$. Furthermore, none of the putative enhancer regions for $C D K N 1 C$ are affected by the deletion (Figure 5b). ${ }^{17}$ The inverted region between the deletions extends over 504.739 kb (hg19, chr11:g.1863937_2368676 inv) and contains about 20 genes, including the imprinted genes IGF2 and H19 together with the ICR1, for which a normal methylation could be detected in the mother and both twins (Figure 5a).

\section{DISCUSSION}

We report on a novel familial deletion in BWS, which is part of a complex structural rearrangement on chromosome $11 \mathrm{p} 15$. In contrast to all other deletions reported for this region so far, the deletion described here does not include the ICR2 itself, but leads to a complete loss of methylation of the ICR2 when maternally inherited.

Strikingly, in this family at least five out of six conceptions showed the same severe phenotype with giant omphalocoeles that were not compatible with life. Although such an outcome is statistically possible, it seems rather unusual. A possible explanation for such a segregation distortion could be that oocytes harbouring the maternal chromosome 11 with the rearrangement have a selective advantage. The reason for this is unclear. One candidate factor is CDKN1C. This protein has been shown to have a role in cell cycle control, but it may also have a role in meiotic progression. Zhang et al. ${ }^{19}$ and Grondahl et $a l^{20}$ have shown that CDKN1C is expressed in human oocytes. However, in familial cases with CDKN1C mutations there is no evidence for segregation distortion. In mice two different models deleting the $5^{\prime}$ part of Cdkn1c have been developed. ${ }^{21,22}$ In both models, offsprings inheriting the deletion from their mother showed a high lethality shortly before or after birth. Most importantly, genotyping between E18.5 an E20 showed a normal distribution of the genotypes. ${ }^{22}$ On the other hand, the transmission ratios in the families with deletions affecting the ICR2 described in the literature so far show a trend towards segregation distortion. In at least three of the six familial cases the deletions seem to be maternally inherited more often as anticipated. In the first family described at least three out of four children were affected. ${ }^{9}$ Additionally, two of two siblings in the second family ${ }^{10}$ and three to four out of five children in the third family ${ }^{7}$ have inherited the deletion from their respective mother. In the three remaining families, two deletions occurred de novo in the patients, ${ }^{7,11}$ and in the last family only one child was reported. ${ }^{8}$ However, the number of such families is small and more data are needed to find out if there is really a segregation distortion in favour of the mutated maternal allele. 
a



normal region

inverted region

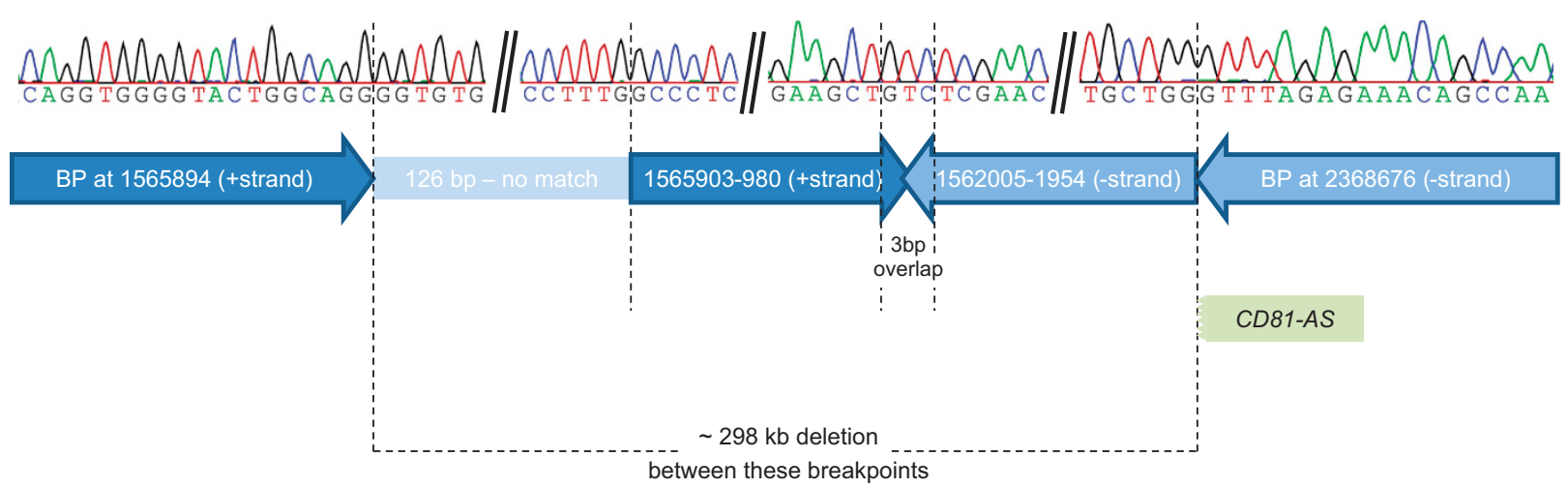

b

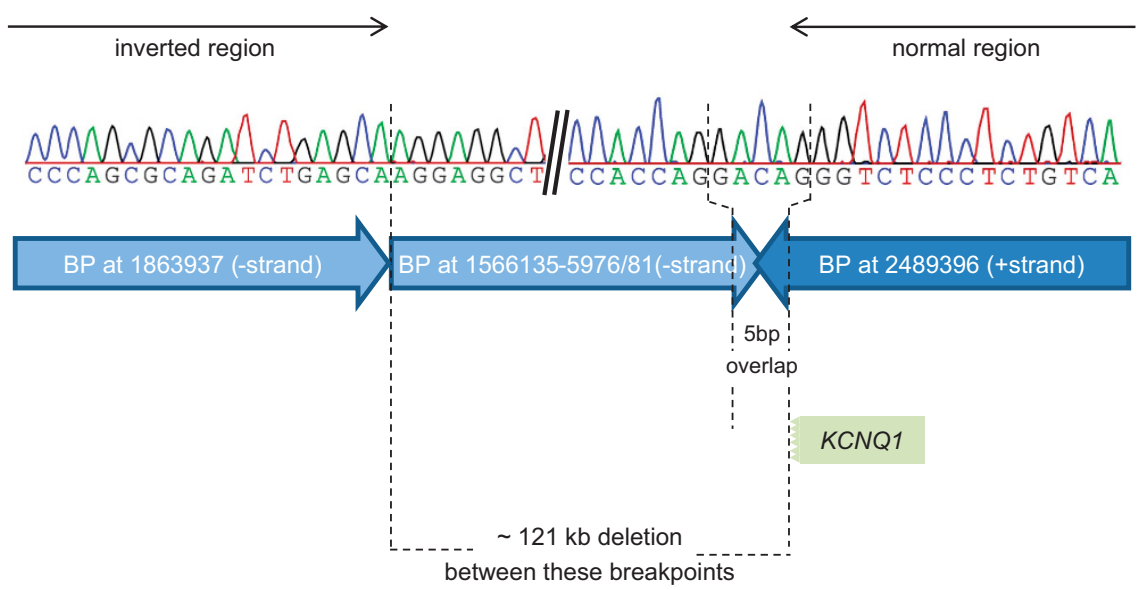

Figure 4 Detailed overview of the breakpoints with Sanger sequencing results. The figure shows the results of the Sanger sequencing of the two breakpoint regions ( $\mathbf{a}$ and $\mathbf{b}$ ) with the corresponding chromosome positions and orientations (based on hg19). The corresponding strand is indicated by the direction and colours of the arrows (dark blue - positive strand; light blue - negative strand). Not drawn to scale.

Of note in this regard is the family described by Demars et al. ${ }^{12}$ harbouring a $\sim 50 \mathrm{~kb}$ duplication within the $5^{\prime}$ part of KCNQ1, which does not affect the ICR2 itself. In this family, four to five of six children had inherited the duplication maternally in the third generation. Strikingly, in the second generation a paternal transmission of the duplication to five out of five children was described, so that a segregation distortion seems to be present after maternal and also after paternal transmission of the duplication. This has not been observed in the families with a deletion described above, but maybe partly due to lack of data. Additionally, a three-generation family with a microduplication including the ICR2 has been described, ${ }^{23}$ where four out of six children of the second generation inherited the duplication paternally. In the third generation, two sisters had five children with Silver-Russel syndrome and only one healthy child, whereas two brothers had six healthy children. Therefore, an effect of the deletion on oocyte growth or meiotic progression seems to be unlikely as there is no explanation why a duplication should act differently than a deletion. It remains unclear whether the observed segregation distortion is a statistical fluke or due to an effect on a pathway that is present in the maternal and the paternal germline alike.

The rearrangement described here leads to the loss of the $5^{\prime}$ part of KCNQ1 but not of the ICR2. Furthermore, the complete loss of methylation on the normally methylated maternal allele points to a failure in imprint establishment in the maternal germline rather than a postzygotic failure in imprint maintenance as is presumably the case in patients with imprinting defects, which is usually present in a mosaic state. ${ }^{1,2}$ In addition, the $C D K N 1 C$ enhancers that have been proposed by Cerrato et al..$^{17}$ are not deleted either. If other, so far unrecognised regulatory elements upstream of KCNQ1 are affected by the deletion or relocated due to the inversion cannot be excluded. It is possible that additional oocyte-specific start sites of KCNQ1 could be affected by the deletion as well, so that no residual expression of KCNQ1 would be present in the oocyte.

Interestingly, in the BWS family with a $\sim 50 \mathrm{~kb}$ duplication of the $5^{\prime}$ part of KCNQ1 on the maternal allele that is also not affecting the ICR2 itself a complete loss of methylation is observed as well. ${ }^{12}$

There is recent evidence for imprinted loci on chromosomes 20 and 15 that transcription through the DMR is required for the establishment of the methylation imprint in the maternal germline. ${ }^{24-26} \mathrm{It}$ is possible that this is also true for the ICR2 on chromosome 11. We therefore suggest that transcription of KCNQ1 through the ICR2 in the maternal germline is required for the imprint establishment in humans (Figure 6). This hypothesis is further supported by results obtained from transgenic mouse lines. ${ }^{27}$ These were generated using BAC clones spanning KCNQ1OT1 and large parts of KCNQ1 of different length but without its promoter. In both cases, the imprint at the ICR2 was not set after germline transmission, presumably caused by the lack of KCNQ1 transcription. 
a

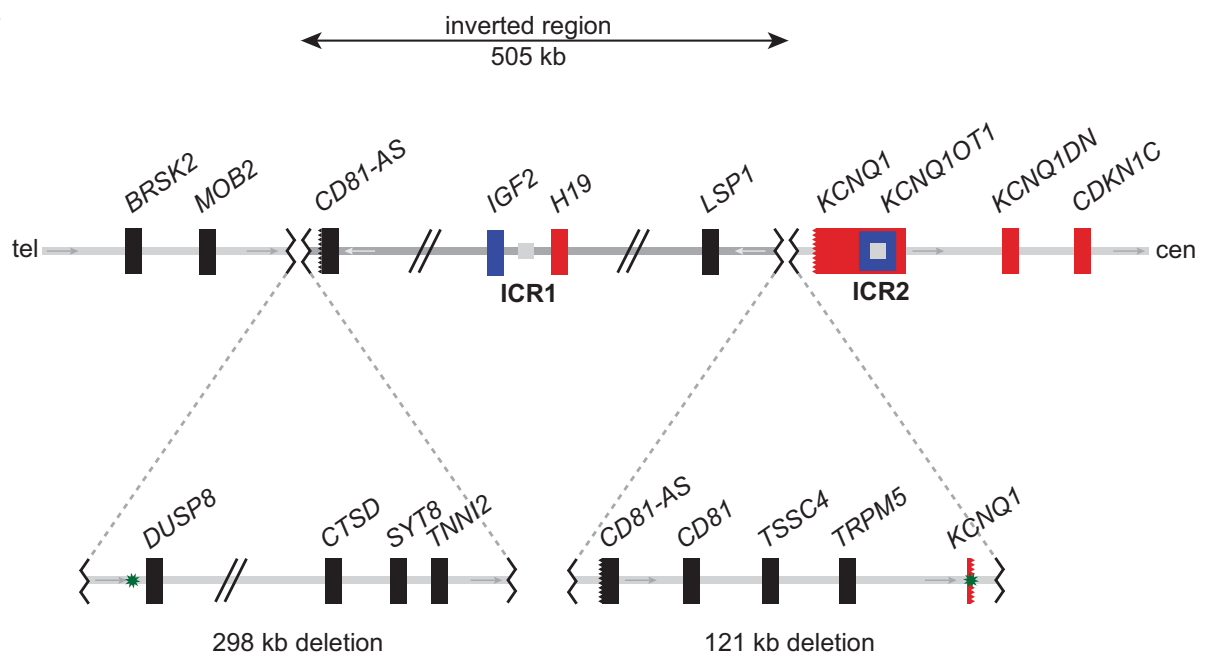

b $K C N Q 1$

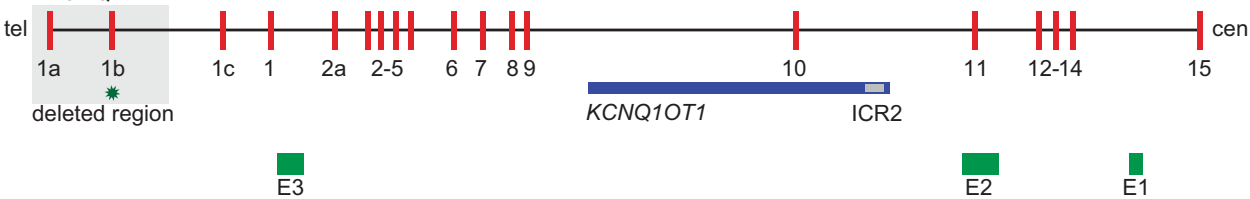

Figure 5 Schematic overview of (a) the rearrangement and (b) of KCNQ1. (a) Schematic overview of the rearrangement based on the Sanger sequencing results of the breakpoint regions. The orientation of the depicted fragments is indicated by arrows. The genes CD81-AS and KCNQ1 are both disrupted by the proximal $121 \mathrm{~kb}$ deletion. Black boxes - biallelically expressed genes; red boxes - maternally expressed genes; blue boxes - paternally expressed genes; tel - telomeric; cen - centromeric. Grey squares indicate the ICRs 1 and 2. Green stars indicate the location of the MSA marker (D11S1984) telomeric to DUSP8. Not drawn to scale. (b) Detailed overview of KCNQ1. The scheme shows the maternally expressed KCNQ1 gene in red, the paternally expressed KCNQ1OT1 in blue, the ICR2 in grey, three putative enhancer regions for CDKN1C according to Cerrato et al. ${ }^{17}$ in green and the deletion affecting the $5^{\prime}$ part of the gene in light grey. The green star indicates the location of the MLPA probe (L02903) within exon 1b of KCNQ1. Exon numbering according to Cerrato et al. ${ }^{17}$ and Neyroud et al. ${ }^{18}$ Not drawn to scale.



Figure 6 Hypothesis model. The situation in the oocyte in relation to KCNQ1 and the ICR2 is given. In a normal oocyte expression of KCNQ1 enables setting of the methylation imprint. The deletion of the $5^{\prime}$ part of $K C N Q 1$ prevents transcription and thus the setting of the methylation imprint in the oocyte. Not drawn to scale.

The deletion described in our family as well as the duplication described by Demars et al..$^{12}$ affect the $5^{\prime}$ part of KCNQ1 and probably interfere with its expression in the oocyte. That KCNQ1 is expressed in human and murine oocytes has already been shown. ${ }^{28,29}$ Furthermore, Chotalia et al..$^{24}$ also detected oocyte-specific start sites for Kcnq1.

The high recurrence of the structural rearrangement at least in the family described here highlights the need for careful data analysis and interpretation in case of a pathogenic molecular genetic finding in the routine diagnostic. Furthermore, a detailed analysis of structural rearrangements as well as fine mapping of deletion and duplication breakpoints is necessary to evaluate the full recurrence risk and to yield further insight into the pathogenesis of imprint regulation at the ICR2 and other imprinted loci.

\section{CONFLICT OF INTEREST}

The authors declare no conflict of interest.

\section{ACKNOWLEDGEMENTS}

We are grateful to the family for their participation in the study, Dagmar Wieczorek for initiating WGS, Nuria Brämswig for her help regarding the WGS 
data and Sabine Kaya, Melanie Heitmann and Christina Lich for expert technical assistance. This work was funded by the Bundesministerium für Bildung und Forschung (BMBF; Imprinting diseases, Grant No. 01GM1513A and D).

1 Weksberg R, Shuman C, Beckwith JB: Beckwith-Wiedemann syndrome. Eur J Hum Genet 2010; 18: 8-14.

2 Eggermann T, Algar E, Lapunzina P et al: Clinical utility gene card for: BeckwithWiedemann Syndrome. Eur J Hum Genet 2014; 22: doi:10.1038/ejhg.2013.132.

3 Eggermann T, Netchine I, Temple IK et al: Congenital imprinting disorders: EUCID.net - a network to decipher their aetiology and to improve the diagnostic and clinical care. Clin Epigenet 2015; 7: 23

4 Choufani S, Shuman C, Weksberg R: Beckwith-Wiedemann syndrome. Am J Med Genet C 2010; 154C: 343-354.

5 Choufani S, Shuman C, Weksberg R: Molecular findings in Beckwith-Wiedemann syndrome. Am J Med Genet C 2013; 163C: 131-140.

6 Begemann M, Spengler S, Gogiel M et al: Clinical significance of copy number variations in the 11 p15.5 imprinting control regions: new cases and review of the literature. J Med Genet 2012; 49: 547-553.

7 Baskin B, Choufani S, Chen YA et al: High frequency of copy number variations (CNVs) in the chromosome $11 \mathrm{p} 15$ region in patients with Beckwith-Wiedemann syndrome. Hum Genet 2014; 133: 321-330.

8 Gurrieri F, Zollino M, Oliva A et al: Mild Beckwith-Wiedemann and severe long-QT syndrome due to deletion of the imprinting center 2 on chromosome 11p. Eur J Hum Genet 2013; 21: 11-969.

9 Niemitz EL, DeBaun MR, Fallon J et al: Microdeletion of LIT1 in familial Beckwith-Wiedemann syndrome. Am J Hum Genet 2004; 75: 844-849.

10 Algar E, Dagar V, Sebaj M, Pachter N: An 11 p15 imprinting centre region 2 deletion in a family with Beckwith-Wiedemann syndrome provides insights into imprinting control at CDKN1C. PLoS One 2011; 6: e29034.

11 Zollino M, Orteschi D, Marangi $G$ et al: A case of Beckwith-Wiedemann syndrome caused by a cryptic $11 \mathrm{p} 15$ deletion encompassing the centromeric imprinted domain of the BWS locus. J Med Genet 2010; 47: 429-432.

12 Demars J, Rossignol S, Netchine I et al: New insights into the pathogenesis of Beckwith-Wiedemann and Silver-Russell syndromes: contribution of small copy number variations to 11 p15 imprinting defects. Hum Mutat 2011; 32: 1171-1182.

13 Kolarova J, Tangen I, Bens S et al: Array-based DNA methylation analysis in individuals with developmental delay/intellectual disability and normal molecular karyotype. Eur J Med Genet 2015; 58: 419-425.

14 Court F, Tayama C, Romanelli V et al: Genome-wide parent-of-origin DNA methylation analysis reveals the intricacies of human imprinting and suggests a germline methylation-independent mechanism of establishment. Genome Res 2014; 24: 554-569.

15 Wieczorek D, Newman WG, Wieland T et al: Compound heterozygosity of low-frequency promoter deletions and rare loss-of-function mutations in TXNL4A causes Burn-McKeown syndrome. Am J Hum Genet 2014; 95: 698-707.

16 Eggermann T, Heilsberg AK, Bens S et al: Additional molecular findings in 11p15associated imprinting disorders: an urgent need for multi-locus testing. $\mathrm{J} \mathrm{Mol} \mathrm{Med}$ (Berl) 2014; 92: 769-777.

17 Cerrato F, De Crescenzo A, Riccio A: Looking for CDKN1C enhancers. Eur J Hum Genet 2014; 22: 442-443.

18 Neyroud N, Richard P, Vignier N et al: Genomic organization of the KCNQ1 K+ channel gene and identification of C-terminal mutations in the long-QT syndrome. Circ Res 1999; 84: 290-297.

19 Zhang P, Kerkela E, Skottman $\mathrm{H}$ et al: Distinct sets of developmentally regulated genes that are expressed by human oocytes and human embryonic stem cells. Fertil Steril 2007; 87: 677-690.

20 Grondahl ML, Yding Andersen C, Bogstad J, Nielsen FC, Meinertz H, Borup R: Gene expression profiles of single human mature oocytes in relation to age. Hum Reprod 2010; 25: 957-968.

21 Yan $\mathrm{Y}$, Frisen J, Lee $\mathrm{MH}$, Massague J, Barbacid $\mathrm{M}$ : Ablation of the CDK inhibitor p57Kip2 results in increased apoptosis and delayed differentiation during mouse development. Genes Dev 1997; 11: 973-983.

22 Zhang P, Liegeois NJ, Wong $\mathrm{C}$ et al: Altered cell differentiation and proliferation in mice lacking p57KIP2 indicates a role in Beckwith-Wiedemann syndrome. Nature 1997; 387: 151-158.

23 Bonaldi A, Mazzeu JF, Costa SS et al: Microduplication of the ICR2 domain at chromosome 11p15 and familial Silver-Russell syndrome. Am J Med Genet A 2011; 155A: 2479-2483.

24 Chotalia M, Smallwood SA, Ruf $\mathrm{N}$ et al: Transcription is required for establishment of germline methylation marks at imprinted genes. Genes Dev 2009; 23: 105-117.

25 Lewis MW, Brant JO, Kramer JM et al: Angelman syndrome imprinting center encodes a transcriptional promoter. Proc Natl Acad Sci USA 2015; 112: 6871-6875.

26 Smith EY, Futtner CR, Chamberlain SJ, Johnstone KA, Resnick JL: Transcription is required to establish maternal imprinting at the Prader-Willi syndrome and Angelman syndrome locus. PLoS Genet 2011; 7: e1002422.

27 John RM, Ainscough JF, Barton SC, Surani MA: Distant cis-elements regulate imprinted expression of the mouse p57( Kip2) (Cdkn1c) gene: implications for the human disorder, Beckwith-Wiedemann syndrome. Hum Mol Genet 2001; 10: 1601-1609.

28 Xue Z, Huang K, Cai C et al: Genetic programs in human and mouse early embryos revealed by single-cell RNA sequencing. Nature 2013; 500: 593-597.

29 Yerushalmi GM, Salmon-Divon M, Yung Y et al: Characterization of the human cumulus cell transcriptome during final follicular maturation and ovulation. Mol Hum Reprod 2014; 20: 719-735.

Supplementary Information accompanies this paper on European Journal of Human Genetics website (http://www.nature.com/ejhg) 